\title{
Preconceitos que se Cruzam: A Relação entre o Racismo, Sexismo e Valores
}

\author{
Ana Karolyne Florencio Amorim ${ }^{1}$ \\ Larisse Helena Gomes Macêdo Barbosa \\ Katia Correa Vione ${ }^{3}$ \\ Olivia Dayse Leite Ferreira ${ }^{4}$ \\ Tailson Evangelista Mariano ${ }^{1}$ \\ Francicléia Lopes Silva ${ }^{1}$ \\ ${ }^{1}$ Universidade Federal da Paraíba, João Pessoa, Paraíba, Brasil \\ ${ }^{2}$ Universidade Cruzeiro do Sul, João Pessoa, Paraíba, Brasil \\ ${ }^{3}$ University of Derby, Derby, Derbyshire, United Kingdom \\ ${ }^{4}$ Universidade Federal do Rio Grande do Norte, Natal, Rio Grande do Norte, Brasil
}

\begin{abstract}
Resumo
Essa pesquisa objetivou analisar a relação entre o Racismo Moderno e o Sexismo Ambivalente utilizando os Valores Humanos como terceira variável que pudesse explicar essa relação, tendo em vista seu poder de predição de fenômenos sociais. A amostra foi composta por 200 participantes distribuídos quase igualmente quanto ao sexo, sendo 101(50,5\%) do sexo feminino e 99 $(49,5 \%)$ do sexo masculino. A média de idade dos respondentes foi de 23 anos $(D P=5,41)$. Observou-se que o Sexismo Ambivalente e o Racismo Moderno estão correlacionados positivamente como também se relacionam com as subfunções Realização, Interativa e Normativa dos Valores Humanos explicando parcialmente a relação entre o racismo e o sexismo. Concluiu-se que as expressões modernas de racismo e sexismo estão interligadas e que os valores normativos, que visam manter a estabilidade social e tradição, podem desempenhar um papel importante na explicação parcial dessa ligação.

Palavras-chave: discriminação sexual, valores sociais, atitudes étnicas e raciais, problemas sociais
\end{abstract}

\section{Prejudices that Cross: The Relationship between Racism, Sexism and Values}

\begin{abstract}
This study aimed to analyze the relationship between Modern Racism and Ambivalent Sexism using Human Values as a third variable that could explain this relationship, given its predictive power against social phenomena. The sample consisted of 200 participants distributed almost equally regarding gender, including 101 (50.5\%) women and 99 (49.5\%) men. The mean age of the respondents was 23 years $(\mathrm{SD}=5.41)$. It was observed that Ambivalent Sexism and Modern Racism are positively correlated as they are also related to the Realization, Interactive, and Normative subfunctions of Human Values, partially explaining the relationship between racism and sexism. It was concluded that modern expressions of racism and sexism are interconnected and that normative values, which aim to maintain social stability and tradition, may play an important role in partially explaining this connection.
\end{abstract}

Keywords: gender discrimination; social values; racial and ethnic attitudes; social issues

Preconceptos que se Cruzan: La Relación entre el Racismo, el Sexismo y los Valores

\section{Resumen}

Esta investigación objetivó analizar la relación entre el Racismo Moderno y el Sexismo Ambivalente utilizando los Valores Humanos como la tercera variable que pudiera explicar esa relación, considerando su poder de predicción de fenómenos sociales. La muestra fue compuesta por 200 participantes distribuidos casi por igual cuanto al sexo, siendo 101 (50, 5\%) mujeres y 99 $(49,5 \%)$ hombres. La edad media de los encuestados fue de 23 años (DS = 5,41). Se observó que el Sexismo Ambivalente y el Racismo Moderno se correlacionaron positivamente, dado que también se relacionaron con las subfunciones Realización, Interactiva y Normativa de los Valores Humanos, explicando parcialmente la conexión entre el racismo y el sexismo. Se concluyó que las expresiones modernas de racismo y sexismo están interconectadas y que los valores normativos, que apuntan a mantener la estabilidad social y la tradición, pueden ejecutar un papel importante en la explicación parcial de esta conexión. Palabras clave: discriminación sexual; valores sociales; actitudes étnicas y raciales; problemas sociales

\section{Introdução}

De acordo com o Instituto de Pesquisa em Economia Aplicada (IPEA, 2017), em uma comparação de dados entre 1995 e 2015, indicadores educacionais revelaram que mulheres negras estão em desvantagem em comparação a mulheres e homens brancos. Especificamente, em 2015, embora a taxa de alfabetização tenha crescido, ainda persiste um diferencial racial importante: 4,9\% das mulheres brancas eram 
analfabetas com 15 anos ou mais de idade; no caso das negras, esse número era o dobro: 10,2\%. Em relação a taxa de desocupação, em 2015, a taxa feminina era de $11,6 \%$ e a dos homens foi de $7,8 \%$. No caso das mulheres negras, a proporção chegou a $13,3 \%$. Os maiores índices foram observados entre as mulheres negras com ensino médio completo ou incompleto: neste grupo, a taxa de desocupação, em 2015, foi 17,4\%. Apesar de, proporcionalmente, o rendimento das mulheres negras ter sido o que mais se valorizou ( $80 \%$ ), enquanto o dos homens brancos foi o que menos cresceu (11\%), é possível observar a manutenção de uma ordem - homens brancos, mulheres brancas, homens negros, mulheres negras - do maior para o menor rendimento. Nesse sentido, pensando nas mulheres negras por meio de recortes de preconceito que elas podem sofrer, elas seriam afetadas não apenas por um tipo de preconceito, mas por dois: o racismo e o sexismo.

Estabelecendo o paralelo entre racismo e sexismo (Southern Student Organizing Committee, s/d, citado por Kerner, 2012), se o racismo justifica ou pressupõe a supremacia de uma raça sobre a outra, o sexismo justifica e pressupõe a supremacia de um sexo sobre o outro. Logo, tais formas de preconceito não são fenômenos exclusivos mutuamente. E juntos eles compreendem sistemas sociais intimamente prejudiciais, distintos e emaranhados de dominação e privilégio em benefício próprio que estruturam a incorporação das desigualdades sociais (Krieger, 2020).

Quanto a expressão desses tipos de preconceito, é importante salientar que esta passou por mudanças ao longo do tempo, especialmente encorajadas pela pressão da legislação antipreconceito (Lima, 2013). Logo, essa expressão tornou-se mais sutil. Dentre as novas formas sutis de racismo, o Racismo Moderno (McConahay, Hardee, \& Batts, 1981) reflete a percepção de que os negros estão recebendo mais do que merecem e violando valores importantes para os brancos (Lima \& Vala, 2004). Já dentre as novas formas de sexismo, o Sexismo Ambivalente se caracteriza como uma expressão hostil (sexismo hostil) e benevolente (sexismo benevolente) (Glick \& Fiske, 1996; 2011).

No entanto, como compreender essas formas de preconceito de maneira intercalada? Swim, Aikin, Hall e Hunter (1995) acreditam que a semelhança entre as novas formas de racismo e sexismo é devida às semelhantes formas de compreensão que se tem dos dois fenômenos. Da mesma forma que o Racismo Moderno se baseia na supressão de expressões flagrantes de racismo, fazendo com que as pessoas acreditem que este não existe mais, já que não é expresso abertamente, essas mesmas pessoas também podem se posicionar da mesma maneira em relação ao sexismo, acreditando que as demandas das mulheres são inadequadas, pois, supostamente, elas não sofrem mais sexismo.

De forma mais sistemática, as teorias interseccionais buscam compreender as desigualdades sociais complexas como interligadas, sendo as intersecções de raça, classe, gênero, sexualidade, entre outras categorias, tanto ideias como ações (Collins, 2017). O termo "interseccional" é creditado à Creenshaw, uma jurista feminista que buscava explicar como a separação entre as categorias gênero e raça, sem levar em consideração a experiência particular das mulheres negras, ocasionava equívocos em julgamentos legais (Nichols \& Stahl, 2019). Sendo assim, o entrelaçamento entre racismo e sexismo, passou a ser compreendido como uma dupla penalização (Lewis \& Grzanka, 2016).

Em uma revisão sistemática de literatura a fim de compreender como a teoria interseccional era aplicada nos estudos de instituições de ensino superior, Nichols e Stahl (2019) analisaram que os estudos compreendiam contextos diversos, como o trabalho, vida familiar e formas de relacionamento. De modo geral, os estudos enfatizavam a importância da interseccionalidade para analisar ambientes que invisibilizam as mulheres negras, como na área das ciências e tecnologias. Análises pessoais das mulheres negras também são focos de análises nos estudos, demonstrando que suas experiências diferem das dos homens brancos, por exemplo. Essa revisão chama a atenção pela baixa quantidade de estudos que empregam métodos estatísticos. Entende-se a importância de todos os métodos, no entanto, modelos estatísticos podem se demonstrar importantes para estudar o fenômeno, em particular, para fins de generalização.

Em relação ao Sexismo Ambivalente e o racismo, McMahon e Kahn (2016) perceberam que basta salientar a categoria raça para que o sexismo benevolente seja mais expresso de forma aparentemente positiva para mulheres brancas em detrimento das mulheres negras e quando se manipula o subtipo sexual (castidade ou promiscuidade), as mulheres negras que fogem do estereótipo esperado culturalmente são mais "recompensadas" com o sexismo benevolente. Percebe-se que quando a categoria raça, além do gênero entra em questão, há uma mudança de padrão na percepção. Quando se leva em consideração o sexismo que é considerado sutil e "benevolente", ele é mais voltado para mulheres brancas. Já quando o aspecto em questão é a sexualidade, 
no qual predomina culturalmente um estereótipo negativo sexualizado das mulheres negras, as que fogem do padrão são recompensadas pelo sexismo considerado "bom". Aqui percebe-se uma hierarquização de subtipos de mulheres que merecem ser "recompensadas" ou não, sendo as mulheres negras merecedoras de recompensa apenas quando não se comportam "mal" para os padrões estereotípicos.

Ao correlacionar o Inventário de Sexismo Ambivalente com a Escala de Racismo Moderno, Glick e Fiske (1996) esperavam uma correlação significativa do Racismo Moderno com o Sexismo Hostil, já que o primeiro é mais relacionado com a hostilidade racial. Como hipotetizado, Sexismo Hostil e a escala Racismo Moderno correlacionaram-se moderadamente $(r=0,44$; $p<0,01)$, ou seja, participantes com um maior nível de atitudes sexistas hostis pontuaram mais na medida de Racismo Moderno. Embora o Racismo Moderno e o Sexismo Benevolente não tenham se correlacionado para os homens, eles foram significativamente correlacionados para as mulheres $(r=0,40 ; p<0,01)$. Isso demonstra como esses tipos de preconceitos podem estar interligados, sendo o sexismo benevolente percebido de forma singular pelas mulheres. No entanto, esse estudo não teve como principal foco uma discussão mais detalhada sobre essa relação.

No contexto brasileiro, os estudos demonstram que, de forma conjunta, o racismo e sexismo acarretam na vida das mulheres negras barreiras no atendimento à saúde (Ferreira, 2016; 2018; Oliveira, 2018; Silva \& Chai, 2018; Werneck, 2016), repercussões negativas na sexualidade e autoestima (Costa, 2018; Miranda \& Silva, 2015) e ao racismo institucional (Silva $\&$ Euclides, 2018). Achados semelhantes se encontram na literatura internacional que tem demostrado que o racismo tem impactos significativos na saúde mental e geral de negros (Paradies et al., 2015; Pieterse, Todd, Neville, \& Carter, 2012). Contudo, são poucos os estudos brasileiros que se situam dentro do terreno da Psicologia Social para explicar essa intersecção e que empregam outros tipos de análises como as análises estatísticas.

Somado a isso, ainda não se pode afirmar com precisão uma relação de dependência do sexismo e do racismo, ou seja, uma variável não poderá ocasionar a outra diretamente. Apesar de essas variáveis terem se mostrado relacionadas em alguns estudos (McMahon \& Kahn, 2018; Silva \& Chai, 2018; Werneck, 2016), não é possível assumir que se uma pessoa for racista, será sexista e vice-versa. Não obstante, as evidências da relação entre sexismo e racismo não devem ser ignoradas, buscando-se encontrar explicações para essa relação, especialmente explicações que estejam no terreno da Psicologia Social. Por exemplo, é possível admitir que uma terceira variável expresse uma melhor relação entre sexismo e racismo, a exemplo da personalidade e dos valores sociais. O presente estudo enfatizou os valores sociais, mais especificamente a Teoria Funcionalista dos Valores Humanos (Gouveia, 1998; 2003) como explicadores da relação entre sexismo e racismo.

Essa teoria propõe que os valores servem a dois tipos de funcionalidade: motivação e orientação. O cruzamento desses dois tipos funcionais resulta em seis subfunções específicas, a saber: subfunção de existência, expressando valores de sobrevivência, estabilidade pessoal e saúde; subfunção de realização, representada por valores de êxito, poder e prestígio; subfunção normativa, caracterizada por valores de obediência, religiosidade e tradição; subfunção suprapessoal, expressa por beleza, conhecimento e maturidade; subfunção experimentação, com valores de emoção, prazer e sexualidade; subfunção interativa, contendo os valores de afetividade, apoio social e convivência (Gouveia, Milfont, Vione, \& Santos, 2015).

Sendo assim, sabendo-se dos efeitos que a combinação do racismo e sexismo podem refletir de forma negativa na saúde mental e física das mulheres negras (Lewis, Williams, Peppers, \& Gadson, 2017), da importância de se estudar o impacto de múltiplas discriminações na saúde mental (Krieger, 2020), bem como da falta de estudos de viés interseccional no âmbito da Psicologia (Lewis \& Grzanka, 2016), especificamente na Psicologia Social, este estudo assume como problemática a relação do racismo e do sexismo. Além disso, para estabelecer uma melhor explicação para tal relação, busca-se compreender fenômenos psicológicos subjacentes a essa relação. Logo, no presente estudo, busca-se analisar a relação entre o Racismo Moderno e o Sexismo Ambivalente, utilizando os Valores Humanos como terceira variável que pode explicar essa relação. Hipotetiza-se que os valores normativos se correlacionem com o sexismo ambivalente e com o racismo, especialmente as subfunções social e pessoal (Formiga, 2010). Ademais, espera-se que os valores expliquem a relação entre o sexismo e o racismo, tendo em vista que esses valores estão relacionados com padrões culturais que prevaleceram durante anos (Gouveia et al., 2015) e que o Racismo Moderno e o Sexismo Ambivalente apresentarão correlação positiva significativa. 


\section{Método}

\section{Amostra}

A pesquisa foi realizada com uma amostra de 200 participantes, universitários e população geral, distribuídos quase igualmente quanto ao sexo, sendo 101(50,5\%) do sexo feminino e $99(49,5 \%)$ do sexo masculino. A média de idade dos respondentes foi de $23 \operatorname{anos}(D P=5,41)$, sendo a idade mínima 18 e a máxima 46 anos.

\section{Instrumentos}

Os instrumentos utilizados na pesquisa foram o Inventário de Sexismo Ambivalente (Glick \& Fisk, 1996), a Escala de Racismo Moderno, o Questionário de Valores Básicos (QVB; Gouveia, 1998; 2003) e um questionário sociodemográfico para caracterizar os respondentes em função da idade e sexo.

O Inventário de Sexismo Ambivalente foi criado por Glick e Fiske (1996) e é composto por 22 itens. Os itens têm como opções de resposta uma escala tipo Likert de quatro pontos, sendo $1=$ Discordo totalmente e 4 = Concordo totalmente, e são baseados em estereótipos acerca de homens e mulheres. Avalia os fatores Sexismo Benevolente (por exemplo, os itens "Mulheres devem ser queridas e protegidas por homens"; "Em catástrofes, mulheres devem ser resgatadas primeiro") e Sexismo Hostil (por exemplo, "Mulheres interpretam ações inocentes como sendo sexistas"; "Feministas procuram que as mulheres tenham mais poder"). A adaptação ao contexto brasileiro foi realizada por Formiga, Gouveia e Santos (2002). Foi observada a existência de um modelo bifatorial conforme o hipotetizado teoricamente. As consistências internas dos dois fatores foram $\alpha=0,77$ para o Sexismo Hostil e $\alpha=0,66$ para o Sexismo Benevolente, sendo consideradas satisfatórias.

A Escala de Racismo Moderno foi criada por McConahay, Hardee e Batts (1981). É composta por 10 itens que representam dois fatores: ameaça aos princípios de igualdade e justiça e negação do preconceito e discriminação. Exemplos de itens correspondentes a esses fatores são, respectivamente: "Eles têm conseguido mais do que merecem" e "Eles não necessitam de ajuda, apenas devem se esforçar". Essa escala foi adaptada ao contexto brasileiro por Santos, Gouveia, Navas, Pimentel e Gusmão (2006). A estrutura bifatorial da escala foi encontrada, porém os autores renomearam os fatores para Negação do Preconceito e Afirmação de Diferenças, pois os acharam mais adequados à realidade brasileira. Os dois fatores apresentaram consistência interna satisfatória ( $\alpha=0,71$ para Negação de Preconceito e $\alpha=0,74$ para Afirmação de Diferenças).

O Questionário de Valores Básicos (QVB; Gouveia, 2003) é composto por 18 itens (exemplos de itens: "Obediência. Cumprir seus deveres e obrigações do dia a dia; respeitar seus pais, os superiores e os mais velhos."; "Poder. Ter poder para influenciar os outros e controlar decisões; ser o chefe de uma equipe."), respondidos em uma escala de 7 pontos, variando de 1 (Nenbuma importância) a 7 (Extremamente importante) de acordo com o grau de importância que cada valor representa na vida das pessoas que os respondem. O QVB apresenta consistência interna equivalente a $\alpha=0,88$.

\section{Procedimentos}

A coleta de dados foi iniciada após a emissão do Parecer Consubstanciado (CAAE: 62356616.3.0000.5181) provido pelo Comitê de Ética de uma Instituição de Ensino Superior. Para cumprir com o procedimento do Consentimento Informado, todos os participantes da pesquisa foram previamente informados de seu objetivo, os riscos, procedimentos, como também seu direito de recusar ou interromper sua participação. A coleta foi realizada de forma presencial e via internet.

Para a coleta de dados presencial (com as escalas impressas), os participantes foram abordados em praças de alimentação ou em salas de aula de uma instituição de nível superior. Para se ter acesso às salas de aula, a pesquisadora entrou em contato com o(a) coordenador(a) do curso, fornecendo explicações acerca da pesquisa para que se pudesse ter a autorização da coleta dos dados nas salas. Após a autorização, aos presentes da sala foi entregue junto aos instrumentos para a coleta o Termo de Consentimento Livre e Esclarecido (TCLE). Para a coleta de dados via internet, os respondentes foram solicitados a responder a pesquisa via e-mail e divulgação em redes sociais. Tanto a forma impressa quanto on-line do questionário teve todas as instruções necessárias para o preenchimento individual dele, sem a necessidade de intervenção das pesquisadoras.

Foram incluídos os indivíduos que concordaram em participar da pesquisa quando foram abordados em sala de aula ou que contatados por e-mail ou redes sociais aceitaram participar da pesquisa e que também eram maiores de 18 anos. Foram excluídos da amostra os indivíduos que não se encaixaram nos padrões descritos acima. 


\section{Análise de Dados}

Os dados referentes ao questionário sociodemográfico, o Inventário de Sexismo Ambivalente, a Escala de Racismo Moderno e o Questionário de Valores Básicos foram avaliados a partir do Statistical Package for the Social Sciences (SPSS) para Windows - versão 21.0. Inicialmente os dados foram submetidos a uma análise descritiva para se ter conhecimento das médias, dos desvios padrões e das pontuações de todas as variáveis. Foram também realizadas análises de correlação $(r$ de Pearson) para analisar se as variáveis estariam relacionadas. Por fim, correlações parciais foram executadas para compreender se os Valores Humanos seriam suficientemente possíveis de explicar o racismo e o sexismo.

\section{Resultados}

Tendo por objetivo proporcionar informações mais detalhadas ao leitor, decidiu-se estruturar os resultados do presente estudo em duas partes principais: (a) Correlação entre os preconceitos e (b) Correlatos valorativos do sexismo e do Racismo Moderno.

\section{Correlação entre os Preconceitos}

O passo seguinte se deu para compreender se o Racismo Moderno e o Sexismo Ambivalente apresentariam correlação positiva significativa. Os valores são dispostos na Tabela 1 .

Como se pode perceber, a correlação entre as variáveis foi positiva e moderada, o que sugere que quanto mais as pessoas são racistas, mais elas são sexistas.

Em relação aos fatores, o fator Afirmação de Diferenças teve uma relação mais forte com o fator Sexismo Benévolo $(r=0,62, p<0,001)$ mostrando que 38,44 $\%$ da variância desse fator é explicada pelo Sexismo Benévolo. O fator Sexismo Hostil relacionou-se positivamente com o fator Negação de Preconceito $(r=$ $0,65, p<0,001)$ tendo $42,25 \%$ da variância explicada pela Negação de Preconceito. O fator Sexismo Benévolo correlacionou-se positivamente com Negação de Preconceito $(r=0,55, p<0,001)$ sendo 30,25\% de sua variância explicada por este último fator. Por fim, Sexismo Hostil também se correlacionou positivamente com Afirmação de Diferenças $(r=0,56, p<$ $0,001)$ com $31,47 \%$ de variância explicada pela Afirmação de Diferenças.

\section{Correlatos Valorativos do Sexismo e do Racismo Moderno}

Relacionando as subfunções dos Valores Humanos com os fatores da Escala de Racismo Moderno e do Inventário de Sexismo Ambivalente, obtiveram-se os valores dispostos na Tabela 2.

As subfunções que apresentaram correlação significativamente com o racismo e o sexismo foram a subfunção Realização, a Interativa e a Normativa. A subfunção Realização apresentou relação positiva $(r=$ $0,14, p<0,05)$ com o fator Negação de Preconceito. Essa mesma subfunção, Realização, também apresentou relação positiva com o fator Sexismo Benévolo $(r$ $=0,15, p<0,05)$. A subfunção Interativa, por sua vez, apresentou correlação negativa com o fator Negação de Preconceito $(r=-0,21, p<0,001)$. Por último, a subfunção Normativa relacionou-se positivamente com o Racismo Moderno $(r=0,23, p<0,001$ para Negação de Preconceito e $r=0,37, p<0,001$ para Afirmação de Diferenças) e o Sexismo Ambivalente $(r=0,51, p<$ 0,001 para Sexismo Benévolo $r=0,40, p<0,001$ para Sexismo Hostil).

Após observar as correlações e a variância compartilhada entre o racismo, o sexismo e os Valores Humanos, uma correlação parcial foi realizada. Utiliza-se correlação parcial para descobrir a variância única da relação entre duas variáveis quando uma terceira variável tem relação com as duas primeiras. Portanto, se racismo, sexismo e valores estão correlacionados, qual seria a relação entre racismo e sexismo, se excluir a variância compartilhada dos valores com essas duas variáveis? As correlações parciais "excluem" da variância entre racismo e sexismo a parte que é comum por causa dos valores. Considerando que a subfunção

Tabela 1.

Correlação entre o Racismo Moderno e o Sexismo Ambivalente

\begin{tabular}{lcc}
\hline Fatores & Sexismo Benévolo & Sexismo Hostil \\
\hline Negação de Preconceito & $0,55^{* *}$ & $0,65^{* *}$ \\
Afirmação de Diferenças & $0,62^{* *}$ & $0,56^{* *}$ \\
\hline
\end{tabular}

Notas.** $p<0,001$.

Psico-USF, Bragança Paulista, v. 26, n. 2, p. 253-263, abr./jun. 2021 
Realização se correlacionou com as dimensões Negação de Preconceito e Sexismo Benévolo, foi realizada uma correlação parcial controlando a influência desta subfunção nas outras duas variáveis. Os resultados são expostos na Tabela 3.

Percebe-se que antes a correlação entre Negação de Preconceito e Sexismo Benévolo era $r=0,55$ (30,25\% de variância), ao controlar a subfunção Realização, essa correlação passou para $r=0,54$ (29, 38\% de variância) o que significa que ela explica $0,87 \%$ dessa relação.

Os coeficientes ao controlar a subfunção Normativa da correlação entre Negação de Preconceito, Sexismo Hostil e Sexismo Benévolo estão apresentados na Tabela 4.

A correlação entre Negação e Sexismo Hostil passou de $r=0,65$ (42,25\% de variância) para $r=0,62$

Tabela 2.

Correlação entre os Preconceitos e os Valores Humanos

\begin{tabular}{|c|c|c|c|c|c|c|c|c|c|c|}
\hline Fatores & 1 & 2 & 3 & 4 & 5 & 6 & 7 & 8 & 9 & 10 \\
\hline $\begin{array}{l}\text { 1.Negação de } \\
\text { Preconceito }\end{array}$ & & & & & & & & & & \\
\hline $\begin{array}{l}\text { 2. Afirmação de } \\
\text { Diferenças }\end{array}$ & $0,53 * *$ & & & & & & & & & \\
\hline 3. Sexismo Benévolo & $0,55^{* *}$ & $0,62^{* *}$ & & & & & & & & \\
\hline 4. Sexismo Hostil & $0,65^{* *}$ & $0,56^{* *}$ & $0,80^{* *}$ & & & & & & & \\
\hline 5. Existência & $-0,13$ & $-0,00$ & $-0,02$ & $-0,04$ & & & & & & \\
\hline 6. Realização & $0,14^{*}$ & 0,06 & $0,15^{*}$ & 0,12 & $0,45^{* *}$ & & & & & \\
\hline 7. Normativa & $0,23 * *$ & $0,37 * *$ & $0,51 * *$ & $0,40 * *$ & $0,45^{* *}$ & $0,41 * *$ & & & & \\
\hline 8.Suprapessoal & $-0,13$ & $-0,09$ & $-0,06$ & $-0,10$ & $0,66^{* *}$ & $0,43 * *$ & $0,26 * *$ & & & \\
\hline 9.Experimentação & $-0,04$ & $-0,01$ & 0,01 & $-0,02$ & $0,45^{* *}$ & $0,36^{* *}$ & $0,18^{*}$ & $0,53 * *$ & & \\
\hline 10.Interativa & $-0,21 * *$ & $-0,01$ & $-0,01$ & $-0,09$ & $0,67 * *$ & $0,40 * *$ & $0,43 * *$ & $0,63 * *$ & $0,45^{* *}$ & \\
\hline
\end{tabular}

Notas. ${ }^{*} p<0,05,{ }^{* *} p<0,001$.

Tabela 3.

Correlação Parcial com Variável de Controle Realização

\begin{tabular}{lll}
\hline Variáveis de controle & Negação & Sexismo Benévolo \\
Realização Negação de Preconceito & & $0,54^{* *}$ \\
Sexismo Benévolo & & \\
\hline
\end{tabular}

Notas. $* * p<0,001$.

Tabela 4.

Correlação Parcial com Variável de Controle Normativa

\begin{tabular}{llll}
\hline Variáveis de controle & Negação & Sexismo Hostil & Sexismo Benévolo \\
\cline { 2 - 4 } Normativa Negação* & & \\
Sexismo Hostil & $0,62^{* *}$ & & \\
Sexismo Benévolo & $0,52^{* *}$ & $0,75^{* *}$ & \\
\hline
\end{tabular}

Notas. *Fator Negação de Preconceito. $p<0,001$. 
(38,94\% de variância), o que indica que a subfunção Normativa explica 3,31\% dessa relação. O fator Negação de Preconceito, por sua vez, correlacionava-se com Sexismo Benévolo por $r=0,55$ (30,25\% de variância) e com a subfunção Normativa controlada, correlaciona-se por $r=0,52(27,04 \%$ de variância), o que demonstra uma redução de $3,21 \%$ da variância compartilhada, sendo esse o valor da contribuição da subfunção Normativa para a correlação.

Os resultados obtidos ao ao controlar mais uma vez a subfunção Normativa da relação entre Afirmação de Preconceito, Sexismo Hostil e Sexismo Benévolo podem ser observados na Tabela 5.

A correlação entre Afirmação de Preconceito e Sexismo Hostil passou de $r=0,56$ (31,47\% de variância) para $r=0,48(23,62 \%)$ indicando uma redução de $7,85 \%$ de variância compartilhada. Já Afirmação de Diferenças com Sexismo Benévolo passou de $r=$ $0,62(38,44 \%)$ para $r=0,53(28,52 \%)$. Assim, a subfunção Normativa contribui com $9,9 \%$ para explicar essa relação.

Em suma, observou-se que a relação entre o Racismo Moderno e Sexismo Ambivalente pode ser parcialmente compreendida pelos Valores Humanos, especialmente pelas subfunções Realização e Normativa. A seguir, busca-se discutir os resultados encontrados.

\section{Discussão}

Diante do objetivo de analisar a relação entre o Racismo Moderno e o Sexismo Ambivalente e os Valores Humanos como terceira variável para possível explicação dessa relação, os resultados demonstraram que os valores explicam parcialmente a conexão entre essas duas variáveis. Além disso, o Sexismo Ambivalente e o Racismo Moderno relacionaram-se. Não obstante, o poder explicativo dos valores foi relativamente baixo, o que deixa aberta a hipótese para outras variáveis de influência, como a personalidade, crenças e atitudes. No entanto, esse resultado não deve ser renunciado, pois, de qualquer modo, os valores explicam parcialmente a relação e, além disso, pode-se observar uma maior influência da subfunção Normativa nessa relação, especialmente no que diz respeito a dimensão Afirmação de Preconceito. Isso pode ser explicado pelo fato de os valores voltados a necessidades materialistas serem preditivos do preconceito (Formiga, 2010; Lins, Lima-Nunes, \& Camino, 2014).

Ademais, ressalta-se que intervenções em Valores Humanos com o objetivo de modificação comportamental tem se demonstrado eficazes (e.g., Ball-Rokeach, Rokeach, \& Grube, 1984). Sendo assim, é possível se pensar em intervenções direcionadas notadamente à subfunção normativa, que parece ter maior impacto no Racismo Moderno e Sexismo Ambivalente. Embora outras intervenções (e.g., mudança de atitudes) sejam válidas, confia-se que reforçar os valores que permitam a redução do preconceito tenha o potencial de produzir mudanças mais estáveis que reforçar atitudes (Rokeach, 1973), por exemplo. Portanto, embora o impacto direto dos valores na explicação da relação entre racismo e sexismo possa parecer pequeno, não se pode descartar como uma possibilidade de intervenção e educação.

Em relação às correlações positivas entre o Sexismo Ambivalente e o Racismo Moderno, esses resultados corroboram os estudos de Glick e Fiske (1996). Contudo, no caso da amostra deste estudo, as correlações apresentaram-se mais fortes do que no estudo original e todos os fatores se relacionaram, não só o Sexismo Hostil, que mensura o caráter hostil do sexismo derivado de crenças de que um sexo é inferior ao outro, com o Racismo Moderno. Isso pode ser explicado pela diferença entre a Escala de Racismo Moderno original e a validada no contexto brasileiro, a qual apresenta o fator Afirmação de Diferenças. O Sexismo Hostil também é explicado pela afirmação de diferenças entre homens e mulheres guiadas por estereótipos sobre estas últimas, sendo essa relação esperada.

Tabela 5.

Correlação Parcial com Variável de Controle Normativa

\begin{tabular}{llll}
\hline Variáveis de controle & Afirmação & Sexismo Hostil & Sexismo Benévolo \\
\cline { 2 - 4 } Normativa Afirmação* & & & \\
Sexismo Hostil & $0,48^{* *}$ & & \\
Sexismo Benévolo & $0,53^{* *}$ & $0,75^{* *}$ & \\
\hline
\end{tabular}

Notas. $*$ Fator Afirmação de Diferenças. ${ }^{*} p<0,001$.

Psico-USF, Bragança Paulista, v. 26, n. 2, p. 253-263, abr./jun. 2021 
Essa correlação das formas de preconceito mostra que suas estruturas se parecem, já que são formas de preconceito moderno, sugerindo que o racismo e o sexismo podem se ligar e formar uma intersecção de preconceitos. Então, ao que se parece, as pessoas podem ser racistas e sexistas concomitantemente, mulheres negras sofreriam os efeitos dessa intersecção, pois pertencem a ambos os grupos alvos desses preconceitos (Creenshaw, 1991; Davis, 2016; Miranda \& Silva, 2015).

Por outro lado, os resultados demonstram que pessoas movidas pela subfunção Interativa dos Valores Humanos são menos propensas a serem racistas e/ ou sexistas evidenciando que quanto mais as pessoas aspiram às necessidades de pertença, amor e afiliação e prezam por estabelecer e manter relações entre as pessoas por meio dos valores de afetividade, apoio social e convivência menos negam o preconceito racial e são menos sexistas hostis.

De diferente modo, pessoas movidas pela subfunção Normativa, regida pelos valores de tradição, religiosidade e obediência, endossam a preservação da cultura e das normas sociais podem ser mais preconceituosas. Não obstante, essa relação era esperada, pois se sabe que essa subfunção tem a capacidade de predizer tipos de preconceito, como o sexismo (Formiga, 2010) e faz sentido na medida em que as crenças acerca dos tipos de preconceito são ligadas à manutenção de estruturas opressoras que legitimam tais formas.

Diante da tentativa de cumprir os objetivos do estudo, algumas limitações foram percebidas. Uma delas refere-se à amostra estudada. $O$ fato de uma parte da amostra ter sido advinda do ambiente universitário pode refletir um viés nas respostas. Hanel e Vione (2016) demonstraram que as populações universitárias não parecem ser tão homogêneas quanto costuma-se assumir, mas se mostram tão heterogêneos quanto a população geral tanto em termos intra quanto interculturais. Apesar disso, amostras com estudantes universitários são comuns em pesquisas, o que gera uma falta de dados referentes a amostras advindas de outras condições. Outra limitação importante a ser discutida, foi a falta do controle da desejabilidade social que pode ter acarretado um viés de resposta.

Sugerem-se mais pesquisas voltadas à uma visão interseccional, que advindas inclusive da Academia, pode dar um aporte a ação social e à mudança (Collins, 2017; Rosenthal, 2016). Além disso, uma abordagem interseccional em Psicologia reconceitualiza o significado das categorias sociais e tenta identificar e compreender os mecanismos pelos quais as desigualdades são criadas e expressas dentro dessas categorias (Else-Quest \& Hyde, 2016). Grande parte dos estudos encontrados prioriza apenas um tipo de preconceito, o que limita a compreensão do fenômeno, tendo em vista que nossa estrutura social permite que várias formas de preconceito estejam envolvidas, como foi demonstrado neste estudo. Pela natureza correlacional deste estudo, sugerem-se mais estudos experimentais manipulando raça, classe e gênero e enfatizando os valores para investigar o processo que resulta na discriminação a esses grupos.

\section{Referências}

Ball-Rokeach, S. J., Rokeach, M., \& Grube, J. W. (1984). The great American values test. New York, NY: Free Press.

Collins, P. H. (2017). Se perdeu na tradução? Feminismo negro, interseccionalidade e política emancipatória. (Bianca Santana, Trad). Parágrafo, 5(1), 7-17. (Artigo original publicado em 2015). Recuperado de http://revistaseletronicas.fiamfaam.br/index. $\mathrm{php} / \mathrm{recicofi} /$ article/view/559

Costa, A. L. S. (2018). Padrões de beleza e racismo na construção da identidade de mulheres negras. Monografia. Universidade Federal do Maranhão, São Luís. Recuperado de https://monografias.ufma. $\mathrm{br} /$ jspui/handle/123456789/2327

Crenshaw, K. (1991). Mapping the Margins: Intersectionality, Identity Politics, and Violence against Women of Color. Stanford Law Review, 43, 12411252. doi: $10.2307 / 1229039$

Davis, A. (2016). Mulheres, raça e classe. Boitempo Editorial.

Else-Quest, N. M., \& Hyde, J. S. (2016). Intersectionality in quantitative psychological research: I. theoretical and epistemological issues. Psychology of Women Quarterly, 40(2) 155-170. doi: 10.1177/0361684316629797.

Ferreira, K. R. D. (2016). Racismo e sexismo em instituições de saúde do DF: Pré-natal, parto e pós-parto de mulheres negras. Sociedade e Estado, 31(3), 885885. Recuperado de https://periodicos.unb.br/ index.php/sociedade/article/view/6176

Ferreira, C. A. A. (2018). Racismo: Uma questão de saúde pública e de gestão na perspectiva de gênero. 
Revista de Gestão em Sistemas de Saúde, 7(2), 143-156. doi: $10.5585 /$ rgss.v7i2.384

Formiga, N. S., Gouveia, V.V., \& Santos, M. N. (2002). Inventário de sexismo ambivalente: Sua adaptação e relação com o gênero. Psicologia em Estudo, 7(1), 103-111. doi:10.1590/S1413-73722002000100013

Formiga, N. (2010). Prioridades valorativas e Sexismo Ambivalente: Predição do sexismo hostil e benévolo baseado nos valores. Revista de Psicologia, 1(1), 105-114. Recuperado de http://www.periodicos. ufc.br/psicologiaufc/article/view/51/50

Glick, P., \& Fisk, S. T. (1996). The Ambivalent Sexism Inventory: Differentiating hostile and benevolent sexism. Journal of Personality and Social Psychology, 70(3), 491-512. doi: 10.1037/0022-3514.70.3.491

Glick, P., \& Fiske, S. T. (2011). Ambivalent Sexism revisited. Psychology of Women Quarterly, 35(3), 530535. doi: 10.1177/0361684311414832

Gouveia, V. V. (1998). La naturaleza de los valores descriptores del individualismo y del colectivismo: Una comparación intra e intercultural (Tese de doutorado).

Departamento de Psicologia Social, Universidade Complutense de Madri, Espanha.

Gouveia, V. V. (2003). A natureza motivacional dos Valores Humanos: Evidências acerca de uma nova tipologia. Estudos de Psicologia, 8(3), 431- 443. doi: 10.1590/S1413-294X2003000300010

Gouveia, V. V., Milfont, T. L., Vione, K. C., \& Santos, W. S. (2015). Guiding actions and expressing needs: on the psychological functions of values. Psyche, 24(2), 1-14. doi:10.7764/psykhe.24.2.884

Hanel, P. H., \& Vione, K. C. (2016). Do student samples provide an accurate estimate of the general public? PloS one, 11(12), 1-10. doi: 10.1371/journal. pone. 0168354

Instituto de Pesquisa Econômica Aplicada (2017). Retrato das desigualdades de gênero e raça. Recuperado de http://www.ipea.gov.br/portal/ images/stories/PDFs/170306_retrato_das_desigualdades_de_genero_raca.pdf

Kerner, I. (2012). Tudo é interseccional? Sobre a relação entre racismo e sexismo. Tavolari, B [trad.] (Obra original publicada 2009). Novos Estudos, 93, 45-58. doi: 10.1590/S0101-33002012000200005
Krieger,N. (2020).Measures of racism, sexism, heterosexism, and gender binarism for health equity research: From structural injustice to embodied harm-an ecosocial analysis. Annual Review of Public Health. doi: 10.1146/annurev-publhealth-040119-094017

Lewis, J. A., \& Grzanka, P. R. (2016). Applying intersectionality theory to research on perceived racism. Em A. N. Alvarez, C. T. H. Liang \& H. A. Neville (Eds.), Cultural, racial, and ethnic psychology book series. The cost of racism for people of color: Contextualizing experiences of discrimination (p. 31-54). American Psychological Association. doi: 10.1037/14852-003

Lewis, J., Williams, M., Peppers, E., \& Gadson, C. (2017). Applying intersectionality to explore the relations between gendered racism and health among Black women. Journal of Counseling Psychology, 64(5):475486. doi: $10.1037 /$ cou0000231

Lima, M. E. O. (2013). Preconceito. Em L. Camino, A. R. R. T. Torres, M. E. O. Lima, M. E. Pereira (Eds.), Psicologia Social: Temas e Teorias. Brasilia: Technopolitik.

Lima, M. E. O., \& Vala, J. (2004). As novas formas de expressão do preconceito e do racismo. Estudos de Psicologia, 9(3), 401-411. doi: 10.1590/ S1413-294X2004000300002

Lins, S. L. B., Lima-Nunes, A. V., \& Camino, L. (2014). O papel dos valores sociais e variáveis psicossociais no preconceito racial brasileiro. Psicologia \& Sociedade, 26(1), 95-105. doi: 10.1590/ S0102-71822014000100011

McConahay, J. B., Hardee, B. B., \& Batts, V. (1981). Has racism declined in America? It depends on who is asking and what is asked. Journal of Conflict Resolution, 25(4), 563-579. doi:10.1177/002200278102500401

McMahon, J. M., \& Kahn, K. B. (2016). Benevolent racism? The impact of target race on ambivalent sexism. Group Processes \& Intergroup Relations, 19(2) 169-183. doi: $10.1177 / 1368430215583153$

McMahon, J. M., \& Kahn, K. B. (2018). When sexism leads to racism: Threat, protecting women, and racial bias. Sex Roles, 78, 591-605. doi: 10.1007/ s11199-017-0828-x

Miranda, M. S., \& Silva, J. L. (2015). As repercussões do racismo na construção da identidade de mulheres negras. Revista Brasileira de Psicologia, 2, 73-84. 
Nichols, S., \& Stahl, G. (2019) Intersectionality in higher education research: A systematic literature review. Higher Education Research \& Development, 38(6), 1255-1268, doi: 10.1080/07294360.2019.1638348

Oliveira, R. J. (2018). A intersetorialidade e a interseccionalidade nas politicas públicas de saúde da mulher negra. (Monografia). Escola Nacional de Administração Pública, Brasília, Distrito Federal. Recuperado de http://repositorio.enap.gov.br/handle/1/3519

Paradies, Y., Ben, J., Denson, N., Elias, A., Priest, N., Pieterse, A., ... \& Gee, G. (2015). Racism as a determinant of health: A systematic review and meta-analysis. PloS one, 10(9).

Pieterse, A. L., Todd, N. R., Neville, H. A., \& Carter, R. T. (2012). Perceived racism and mental health among Black American adults: A meta-analytic review. Journal of Counseling Psychology, 59(1), 1-9.

Rokeach, M. (1973). The nature of human values. New York: Free Press.

Rosenthal, L. (2016). Incorporating intersectionality into psychology: An opportunity to promote social justice and equity. American Psychologist, 71(6), 474-485. doi: 10.1037/a0040323

Santos, W. S., Gouveia, V. V., Navas, M. S., Pimentel, C. E. \& Gusmão, E. E. S. (2006). Escala de
Racismo Moderno: Adaptação ao contexto brasileiro. Psicologia em Estudo. 11. 637-645. doi: 10.1590/ S1413-73722006000300020.

Silva, I. P. A., \& Chai, C. G. (2018). As relações entre racismo e sexismo e o direito à saúde mental da mulher negra brasileira. Revista de Políticas Públicas, 22, 987-1005. Recuperado de http://www.periodicoseletronicos.ufma.br/index.php/rppublica/ article/view/9830/5784

Silva, J., \& Euclides, M. S. (2018). Falando de gênero, raça e educação: Trajetórias de professoras doutoras negras de universidades públicas dos estados do Ceará e do Rio de Janeiro (Brasil). Educar em Revista, 34(70), 51-66. doi: 10.1590/0104-4060.58760

Swim, J. K., Aikin, K. J., Hall, W. S., \& Hunter, B. A. (1995). Sexism and racism: Old-fashioned and modern prejudices. Journal of personality and social psychology, 68(2), 1- 20. doi: 10.1037/0022-3514.68.2.199

Werneck, J. (2016). Racismo institucional e saúde da população negra. Revista Saúde e Sociedade, 25(3), 535-549. doi: 10.1590/S0104-129020162610

Recebido em: 30/04/2019

Reformulado em: 17/05/2020

Aprovado em: 10/08/2020 
Sobre os autores:

Ana Karolyne Florencio Amorim é Graduada em Psicologia pelas Faculdades Integradas de Patos e mestra e doutoranda pela Universidade Federal da Paraíba através do Programa de Pós-Graduação em Psicologia Social. Integra o Grupo de Pesquisa em Comportamento Político, pesquisando sobre sexismo, racismo e legitimidade das desigualdades sociais.

ORCID: https://orcid.org/0000-0002-6104-4512.

E-mail: karollyne_amoriim@hotmail.com

Larisse Helena Gomes Macêdo Barbosa é Doutora em Psicologia Social pela Universidade Federal da Paraíba e Mestra em Psicologia Social na mesma universidade. Graduada em Psicologia pela Universidade Federal da Paraíba. Atualmente, é professora da Universidade Cruzeiro do Sul e tem trabalhado, principalmente, com as áreas de Pesquisa, Análise de Dados, Psicologia Social, Psicologia positiva e Psicometria.

ORCID: https://orcid.org/0000-0002-2965-5540

E-mail: larissehelena@hotmail.com

Katia Correa Vione é Doutora em Psicologia pela Cardiff University, Mestra em Psicologia Social e Graduada em Psicologia pela Universidade Federal da Paraíba. É colaboradora do grupo de Estudos e Práticas em Psicologia Positiva (GEPPsi+) da Universidade Federal do Espírito Santo e do Núcleo de Avaliação Psicológica em Saúde (NapsiS) da Universidade Federal do Ceará. Possui interesse nas áreas de Psicometria, Psicologia Social, Avaliação Psicológica, Metodologia e Estatística e Psicologia Positiva. Atualmente é professora da University of Derby.

ORCID: https://orcid.org/0000-0001-6633-8520

E-mail:katiavione@gmail.com

Olívia Dayse Leite Ferreira possui graduação em Psicologia pela Universidade Federal da Paraíba (2010), mestrado em Psicologia pela Universidade Federal do Rio Grande do Norte (2014) e atualmente é doutoranda do Programa de Ciências da Saúde da Universidade Federal do Rio Grande do Norte. Tem experiência na área de Psicologia clínica (terapia cognitiva-comportamental), neuropsicologia e neurociências, com ênfase nos processos cognitivos.

ORCID: https://orcid.org/0000-0001-5298-8805

E-mail: oliviadayse@yahoo.com.br.

Tailson Evangelista Mariano é Doutorando e Mestre em Psicologia Social pela Universidade Federal da Paraíba e Graduado em Psicologia pela Universidade Federal do Piauí. Atualmente integra o Laboratório de Psicologia da Mídia (LPM), coordenado pelo Professor Dr. Carlos Eduardo Pimentel. Alguns interesses em pesquisas são: efeitos cognitivos e sociais de jogos virtuais, valores humanos, psicometria e avaliação psicológica.

ORCID: https://orcid.org/0000-0001-6716-0250

E-mail: tailsonmariano@hotmail.com

Francicléia Lopes Silva é especialista em Avaliação Psicológica pelas Faculdades Integradas de Patos - FIP e possui graduação em Psicologia pela mesma universidade. Mestra e Doutoranda em Psicologia Social pela UFPB. Atualmente desenvolve pesquisas nas áreas de violência sexual e avaliação psicológica.

ORCID: https://orcid.org/0000-0002-1967-2830

E-mail: francicleia.psi@hotmail.com

Contato com os autores:

Ana Karolyne Florencio Amorim

Rua Antônio Miguel Duarte, 50, Bancários

Residencial dos Sombreiros, apto 204, bloco D.

CEP: 58051-125

Psico-USF, Bragança Paulista, v. 26, n. 2, p. 253-263, abr./jun. 2021 